\title{
Bushen recipe and its disassembled prescriptions inhibit inflammation of liver injury associated with Concanavalin A through Toll-like receptor 3/9 signaling pathway
}

\author{
HONGMING NIE $^{1 *}$, ZHAOHE MEI $^{2 *}$, RONG WANG ${ }^{3 *}$, BINBIN ZHAO $^{2}$, \\ YUEQIU GAO $^{1}$, JIANJIE CHEN $^{1}$ and LINGTAI WANG ${ }^{1}$ \\ ${ }^{1}$ Department of Liver Disease, Shuguang Hospital Affiliated to Shanghai University of Traditional Chinese Medicine; \\ ${ }^{2}$ Department of Traditional Chinese Medicine, Shanghai University of Traditional Chinese Medicine; \\ ${ }^{3}$ Department of Pathology, Shanghai Pudong New Area Traditional Chinese Medicine Hospital, Shanghai 201203, P.R. China
}

Received May 17, 2017; Accepted March 27, 2018

DOI: $10.3892 / \mathrm{mmr} .2018 .9082$

\begin{abstract}
The aim of the present study was to explore the effect of Bushen recipe and its disassembled prescriptions on liver injury and chronic hepatitis B. Liver injury was induced in normal and hepatitis B virus (HBV)-transgenic mice through injection of Concanavalin $\mathrm{A}$, followed by treatment with Bushen recipe and its disassembled prescriptions including the Bushen-yang, the Bushen-yin and the QingHua groups as well as the GanYanLing group (positive control). Subsequently, their liver function indexes were investigated by a microplate method and liver sections were blindly evaluated using an optical microscope by a pathologist. Subsequently, the activation state of Toll-like receptor (TLR)3/9 signaling pathway in liver tissues was analyzed by western blotting. Additionally, the inflammatory factors produced following liver injury in peripheral blood were detected via ELISA. Following intervention with the Bushen recipe and its disassembled prescriptions, the liver function indexe alanine aminotransferase had declined, whereas cholinesterase increased. The pathological alterations of liver tissue in HBV transgenic mice were reversed by Bushen recipe and its disassembled prescriptions. In addition, the TLR3/9 signaling pathway in liver tissues of HBV transgenic mice was inhibited and inflammatory factors such as interleukin (IL)-6, IL-1, tumor necrosis factor- $\alpha$ and interferon- $\gamma$ were reduced significantly.
\end{abstract}

Correspondence to: Dr Hongming Nie or Dr Yueqiu Gao, Department of Liver Disease, Shuguang Hospital Affiliated to Shanghai University of Traditional Chinese Medicine, 528 Zhangheng Road, Shanghai 201203, P.R. China

E-mail: beining0630@126.com

E-mail: gaoyueqiu@hotmail.com

${ }^{*}$ Contributed equally

Key words: Toll-like receptor, hepatitis B virus, Concanavalin A, Bushen recipe, Toll-like receptor 3/9 signaling pathway
In conclusion, the present study demonstrated that Bushen recipe and its disassembled prescriptions repaired liver injury induced by Concanavalin A through inhibition of TLR3/9 signaling pathway.

\section{Introduction}

Infection with hepatitis B virus (HBV) is a public health issue worldwide (1). Epidemiological studies have estimated that 350 million people are chronic carriers of $\mathrm{HBV}$, with the potential to develop acute and chronic hepatitis, which is a major risk factor for the development of hepatocellular carcinoma $(2,3)$. Standard treatment regimens such as pegylated interferons (IFNs) and nucleoside/nucleotide analogs are used to treat chronic hepatitis B but are only partially successful (4-7). Drug resistance and severe side effects limit current therapies for chronic HBV infection (8).

The role of adaptive immune responses in the control of HBV infection have been extensively investigated. Toll-like receptors (TLRs) accept pathogen-associated molecule patterns, and activate antiviral mechanisms including intracellular antiviral pathways and the production of antiviral effectors like IFNs and pro-inflammatory cytokines. Activation of the TLR3 signaling is necessary for the induction of liver damage induced by Concanavalin A in vivo; TLR3 regulates inflammation and the adaptive $\mathrm{T}$ cell immune response in the absence of viral infection (9). A previous study indicated that the ubiquitin proteasome pathway regulates the HBV life cycle through TLR signaling pathway (8). The ubiquitin proteasome system (UPS) is a conserved cellular signaling pathway that controls several critical processes in the cell by regulating expression of the proteins involved in cell cycle, DNA repair, innate immunity, and other processes (2). The UPS mediates the majority of protein degradation in eukaryotic cells preventing accumulation of damaged, misfolded and mutant proteins via proteolysis $(10,11)$. The UPS serves an important role in the cellular response to multiple stimulation signals. Although the primary function of ubiquitin was associated with proteolysis, it is now considered as a key regulator of functions such as signaling cascades, transcription, apoptosis or oncogenesis. 
Malfunction of the UPS is associated with the development of multiple pathological processes including metabolic disorders, immune diseases, inflammation and cancer (12).

Traditional Chinese medicine has served a role in the prevention of hepatitis $\mathrm{B}$ in China. The characteristics of Chinese medicine with its multi-links and multi-pathways are increasingly demonstrating great therapeutic potential and development prospects. Bushen recipe used in traditional Chinese medicine and is mainly comprised of herba epimedii, cuscuta chinensis lam and radix rehmanniae recen, is used to tonify kidneys. However, many Chinese studies recently demonstrated that Bushen recipe is effective for the treatment of numerous diseases such as early stage diabetes, osteoporosis, osteosarcoma and chronic liver disease and our research group previously demonstrated another important efficacy on HBV (13-16). Furthermore, our previous study indicated that not only the whole prescription of Bushen Recipe, but also its disassembled prescriptions may exhibit certain efficacy on liver injury to an extent (Nie et al, unpublished data). It was suggested that Bushen Recipe and its disassembled prescriptions have a bi-directional regulatory effect on ubiquitin ligase, and the ubiquitination of myeloid differentiation primary response (MyD) 88 is affected by the ubiquitin proteasome pathway, which can inhibit the production of inflammatory mediator and activate TIR-domain-containing adapter-inducing interferon- $\beta$ (TRIF) and tumor necrosis factor (TNF) receptor-associated factor-binding kinase (TBK)1. Ultimately the ubiquitin proteasome signaling pathway could serve a dual effect as an anti-inflammatory and inhibitor of replication of hepatitis B virus. In the present study, through simulation of the liver injury induced by viral hepatitis attacks by Concanavalin A treatment, it was verified that Bushen prescription exerted an anti-inflammatory and protective effect on the liver through the multi-link regulation of immunity.

\section{Materials and methods}

Materials. A total of 60 normal Balb/c mice were purchased from Shanghai Jiesijie Experimental Animals Co., Ltd. (Shanghai, China) and $40 \mathrm{HBV}$ transgenic mice were obtained from the Guangzhou 458th Hospital of Chinese People's Liberation Army (Guangzhou, China). Concanavalin A was purchased from Sigma-Aldrich (Merck KGaA, Darmstadt, Germany). The primary antibodies used in the western blot analysis were as follows: Anti-TLR4 (cat. no. NB100-56566), anti-TLR3 (cat. no. NB100-56571) and anti-TLR9 (cat. no. nbp2-24729), purchased from Novus Biologicals LLC (Littleton, CO, USA); anti-TNF-associated factor (TRAF)6 (cat. no. ab33915), anti-IFN regulatory factor (IRF)3 (cat. no. ab25950), anti-TBK1 (cat. no. ab109735), anti-TRAF-interacting protein (TIRP) (cat. no. ab205100) purchased from Abcam (Cambridge, UK); anti-nuclear factor (NF)- $\mathrm{B}$ (cat. no. 8242), anti-MyD88 (cat. no. 4283) purchased from Cell Signaling Technology, Inc. (Danvers, MA, USA). The secondary antibodies were obtained from R\&D Systems, Inc. (Minneapolis, MN, USA). ELISA kits to detect interleukin (IL)-6, IL-1, TNF- $\alpha$ and IFN- $\gamma$ were purchased from Wuhan Huamei Biotech Co., Ltd. (Wuhan, China). Alanine aminotransferase Assay Kit (cat. no. C009-2) and
Cholinesterase assay kit (cat. no. A023-2) were purchased from Nanjing Jiancheng Bioengineering Institute (Nanjing, China).

Animal experiments. The experimental mice were fed in Specific Pathogen Free Animal Rooms of Animal Experimental Center of Shanghai University of Traditional Chinese Medicine (Shanghai, China) with free access to clean drinking water and food. All animal experiments were carried out according to the regulations of and approved by Laboratory Animal Administration of Shanghai University of Traditional Chinese Medicine. All the mice were divided into two groups: Group 1 and group 2. The group 1 were all normal and the group 2 were all HBV transgenic mice: A replicative HBV transgenic mouse produced by the Department of Genetics Engineering, Infectious Disease Center, Guangzhou 458th Hospital of Chinese People's Liberation Army (Guangzhou, China). The HBV genome was transferred into the genotype of 1.3 times $\mathrm{D}$ genotype by microinjection of fertilized eggs, serum HBs Ag >2,000 U/ml, HBV DNA levels of $10^{4}-10^{6}$ copies $/ \mathrm{ml}$, HBV replication intermediates exist in the liver. All the experimental mice were germ-free grade, 6-8 weeks old, weighing 20-22 g, and purchased following testing for HBs Ag. Animals of both sexes (males/females 1:1) were included. Mice in both groups were divided into seven sub-groups: A, Control; B, Concanavalin A; C, Bushen recipe (BS); D, Bushen-yang (BSY); E, Bushen-yin (BSC); F, QingHua (QH); G, GanYanLing (GYL). Each sub-group in group 1 had 8 mice, while 5 mice in each sub-group in group 2; the remaining mice were not employed. Acute liver injury was induced using tail vein injection of Concanavalin $\mathrm{A}$ in both normal and HBV transgenic mice from B to G sub-groups and the mice in each sub-group were treated with different traditional Chinese medicines (diluted in saline), respectively: A, (Control): Normal Saline; B, (Concanavalin A): with Normal Saline, $15 \mathrm{mg} / \mathrm{kg}$; C, (BS): the Whole Bushen recipe; D, (BSY): the disassembled prescriptions of Bushen recipe; $\mathrm{E},(\mathrm{BSC})$ : the disassembled prescriptions of Bushen recipe; $F,(Q H)$ : The disassembled prescriptions of Bushen recipe; G, (GYL).

These traditional Chinese medicines were prepared in our laboratory or were purchased from different companies and their effective constituents and manufacturer were enumerated as follows:

The Whole Bushen recipe (BS) was prepared according to the formula of 'Bushen Granule' (Shanghai Pharmacy) which was produced in our laboratory and is comprised of $15 \mathrm{~g}$ herba epimedii, $15 \mathrm{~g}$ cuscuta chinensis lam, $15 \mathrm{~g}$ cistanche deserticola ma, $15 \mathrm{~g}$ fallopia multiflora, $15 \mathrm{~g}$ radix rehmanniae recen, $15 \mathrm{~g}$ lyceum barbaruml, $15 \mathrm{~g}$ giant knotweed rhizome, $15 \mathrm{~g}$ radix scutellariae, $15 \mathrm{~g}$ radix sophorae flavescentis and $10 \mathrm{~g}$ pericarpium citri reticulatae viride.

The Bushen-yang group (BSY) of Bushen recipe was boilfree particles of Chinese medicine and comprised of $15 \mathrm{~g}$ herba epimedii, $15 \mathrm{~g}$ cuscuta chinensis lam, $15 \mathrm{~g}$ cistanche deserticola ma which was produced by Shenzhen Resources Sanjiu Modern Chinese Medicine Co., Ltd. (Shenzhen, Guangdong, China).

The Bushen-yin group (BSC) of Bushen recipe was also boil-free particles of Chinese medicine and comprised of $15 \mathrm{~g}$ fallopia multiflora, $15 \mathrm{~g}$ radix rehmanniae, $15 \mathrm{~g}$ lyceum barbaruml that was produced by Shenzhen Resources Sanjiu Modern Chinese Medicine Co., Ltd. 
The QingHua group $(\mathrm{QH})$ of Bushen Recipe has $15 \mathrm{~g}$ giant knotweed rhizome, $15 \mathrm{~g}$ radix scutellariae, $15 \mathrm{~g}$ radix sophorae flavescentis and was produced by Shenzhen Resources Sanjiu Modern Chinese Medicine Co., Ltd. These were all produced as boil-free particles of Chinese medicine.

GYL was purchased from Changshu Lei Yun Shang Pharmaceutical Co., Ltd (Changshu, China) and had $35 \mathrm{mg}$ matrine in $2 \mathrm{ml}$ solution of GYL.

The administration dose of these drugs was calculated according to the human and mice dose conversion method in the second edition of 'Experimental Zoology'. The conversion coefficient between human $(\sim 70 \mathrm{~kg})$ and mice $(\sim 20 \mathrm{~g})$ is 0.0026 . Assuming the dose for human $(70 \mathrm{~kg})$ is $\mathrm{n}$, the dose for mice may be calculated as: $\mathrm{n} \mathrm{mg} / \mathrm{kg}$ x $70 \mathrm{~kg}$ x0.0026/20 g=9.1n $\mathrm{mg} / \mathrm{kg}$. Therefore, the dosage which was used in this study was 9 times of the adult daily dose. The mice in sub-groups A to F underwent intragastric administration, while intraperitoneal injection occurred for sub-group $\mathrm{G}$. The drug was administered once daily for 7 days at a dose of $18 \mathrm{mg} / \mathrm{kg}$.

Microplate method to detect liver function indexes. The mice were sacrificed by excessive ether inhalation at $18 \mathrm{~h}$ after injection with Concanavalin A and administration with Bushen recipe and its disassembled prescriptions. Peripheral blood samples $(0.5 \mathrm{ml})$ were collected from hearts of mice and centrifuged at $375 \mathrm{x} \mathrm{g}$ for $30 \mathrm{~min}$ at $4^{\circ} \mathrm{C}$ to obtain serum for the following experiments. Subsequently, serum was seeded into 96-well plates and detection reagents were added according to the manufacturer's protocol of Nanjing Jiancheng Bioengineering Institute (Nanjing, China). Finally, these plates were analyzed at a wavelength of $505 \mathrm{~nm}$ by a Thermo Scientific Varioskan Flash (Thermo Fisher Scientific, Inc., Waltham, MA, USA) to detect the content of alanine aminotransferase (ALT) and cholinesterase (ChE).

Hematoxylin and eosin staining. Mice were sacrificed at $18 \mathrm{~h}$ following injection with Concanavalin A and administration with Bushen recipe and its disassembled prescriptions, liver tissues of all mice were obtained to inspect liver injury and antiviral effect of Bushen recipe and its disassembled prescriptions, and they were fixed with $10 \%$ paraformaldehyde. Subsequently, the paraffin-embedded sections were prepared for histological analysis. The slices were cut in $5 \mu \mathrm{m}$ sections and stained in dye liquor of hematoxylin $(3 \mathrm{~min})$ and eosin $(15 \mathrm{sec})$ at room temperature for histological evaluation. Finally, these slices were observed under an inverted microscope (ix73, Olympus Corporation, Tokyo, Japan) and evaluated by a pathologist blindly. A total of five random fields of hepatic lobule were imaged at x200 magnification.

Western blot analysis. Liver tissues of all mice were homogenized and cells were suspended in PBS. The cell suspension was centrifuged with PBS at $600 \mathrm{x} \mathrm{g}$ at $4^{\circ} \mathrm{C}$ and were lysed with radioimmunoprecipitation assay lysis buffer (Beyotime Institute of Biotechnology, Jiangsu, China) for $30 \mathrm{~min}$ at $4^{\circ} \mathrm{C}$. Subsequently, cell lysates were centrifuged at 9,000 x g at $4^{\circ} \mathrm{C}$ for $15 \mathrm{~min}$ and a Takara BCA Protein Assay Kit (cat. no. T9300A; Takara Bio, Inc., Otsu, China) was used to detect the total protein concentrations. Subsequently, total protein was mixed with an equal volume of loading buffer and boiled for $5 \mathrm{~min}$. Equal amounts of proteins $(20 \mu \mathrm{g})$ in each lane were separated by $10 \%$ SDS-polyacrylamide gel electrophoresis and then transferred to Immobilon-polyvinylidene fluoride Transfer Membranes (EMD Millipore, Billerica, MA, USA). Membranes were blocked with 5\% nonfat milk in TBS with Tween 20 (TBST) for $1 \mathrm{~h}$ at room temperature and incubated with the indicated primary antibodies overnight at $4^{\circ} \mathrm{C}$ in a dilution of $1: 1,000$. Following washing 3 times with TBST, these membranes were incubated with secondary antibodies, including $\operatorname{IgG}(\mathrm{H}+\mathrm{L})$ horseradish peroxidase (HRP)-labeled goat anti-mouse IgG (cat. no. A0216) and $\operatorname{IgG}(\mathrm{H}+\mathrm{L})$ HRP-labeled goat anti-rabbit IgG (cat. no. A0208), purchased from Beyotime Institute of Biotechnology, for $1 \mathrm{~h}$ at room temperature at a dilution of 1:10,000. GAPDH served as the loading control. Finally, membranes were visualized using the enhanced chemiluminescence system (ECL; PerkinElmer Inc., Waltham, MA, USA).

ELISA analysis. Mouse IL-6 ELISA kit (cat. no. EK0411) and mouse TNF- $\alpha$ ELISA kit (cat. no. EK0527) were purchased from Boster (Hubei, China). Mouse IFN- $\gamma$ ELISA kit was purchased from R\&D Systems, Inc. (cat. no. MIF00). Mouse IL-1 ELISA kit was purchased from Mlbio (cat. no. ml037875, Shanghai, China). ELISA analysis in the present study was conducted according to the manufacturer's protocol of Wuhan Sanying Bioengineering (Wuhan, China). In brief, blank wells without any solution were set in a 96-well plate and $100 \mu \mathrm{l}$ negative control (PBS with $0.05 \%$ Tween-20), positive control or serum from rats of the aforementioned experimental groups per well were added into the test wells. The plate was covered with the adhesive strip and incubated in a humidified atmosphere of $5 \% \mathrm{CO}_{2}$ at $37^{\circ} \mathrm{C}$ for $1 \mathrm{~h}$. Subsequently, $50 \mu \mathrm{l}$ of HRP-conjugated antibodies were added into each well (apart from the blank well). Subsequently, plates were covered with another adhesive strip and incubated for $30 \mathrm{~min}$ at $37^{\circ} \mathrm{C}$ again. Following this, wells were washed with wash buffer (included in kit; $200 \mu 1$ per well) three times and were left to dry for $20 \mathrm{sec}$ completely removing the liquid. Following washing, any remaining wash buffer was removed through inverting the plate on clean paper towels. In addition, $50 \mu \mathrm{l}$ substrate $\mathrm{A}$ and $50 \mu \mathrm{l}$ substrate $\mathrm{B}$ were added to each well and plates were incubated for $30 \mathrm{~min}$ at $37^{\circ} \mathrm{C}$ in the dark. Finally, $50 \mu \mathrm{l}$ of stop solution was added into each well, and plates were gently tapped to mix the solution. Finally, optical density (OD) of each well was determined within $10 \mathrm{~min}$ $\left(25^{\circ} \mathrm{C}\right)$ using a Thermo Scientific Varioskan Flash (Thermo Fisher Scientific, Inc.) at a wavelength of $450 \mathrm{~nm}$.

Statistical analysis. All experiments in the present study were repeated at least three times. All data are presented as the mean \pm standard deviation. Data were analyzed by SPSS software, version 18.0 (SPSS, Inc., Chicago, IL, USA). Two-way analysis of variance and Student-Neuman-Keuls method were used to determine statistical significance among experimental groups. $\mathrm{P}<0.05$ was considered to indicate a statistically significant difference.

\section{Results}

Bushen recipe and its disassembled prescriptions inhibit liver injury by affecting liver function indexes. ALT and ChE are 
both synthesized in liver and may reflect the degree of liver injury. Following injection of Concanavalin A, liver tissues of all mice presented liver injury and the Bushen recipe and its disassembled prescriptions have previously been demonstrated to decrease liver injury. Therefore, the expression levels of ALT and ChE were investigated in peripheral blood of normal and HBV transgenic mice following treatment with Concanavalin A, Bushen Recipe and its disassembled prescriptions. As presented in Fig. 1, the results demonstrated that the content of ALT was increased immediately following administration of Concanavalin A, whereas the ChE declined in the liver tissues of both normal and HBV transgenic mice compared with the control. However, this increase of ALT was suppressed following administration of Bushen Recipe and its disassembled prescriptions (Fig. 1A), while the expression of ChE was upregulated (Fig. 1B). These results indicated that Bushen Recipe and its disassembled prescriptions alleviated the liver injury induced by Concanavalin A as observed within the Bushen Recipe and disassembled prescription groups compared with in the Con A group.

Bushen recipe and its disassembled prescriptions repair liver injury induced by Concanavalin A. Livers of mice demonstrated acute immune hepatitis following injection of Concanavalin A. The results obtained in this experiment are presented in Fig. 2. Liver cells of normal mice were well-formed and the structure of hepatic cord was clear and intact. The hepatic lobule had no necrosis (which is a form of cell injury resulting in the premature death of cells in living tissue by autolysis), bleeding, and infiltration of inflammatory cells. However, the opposite phenomenon was observed in the sections of mice injected with Concanavalin A, whereas Bushen recipe and its disassembled prescriptions relieved the pathological features in the HBV transgenic mice, for example, the hepatic necrotic area was significantly decreased in the Bushen recipe and its disassembled prescriptions treated groups. The results indicated that Bushen recipe and its disassembled prescriptions relieved the liver injury induced by Concanavalin A.

Bushen recipe and its disassembled prescriptions repair liver injury through inhibition of TLR3/9 signaling pathway. Protein expression levels of MyD88, TBK1, TRAF6, IRF3, TLR3, TLR9, TLR4, NF-кB and TIRP that are members of TLR3/9 signaling pathway in all mice were detected by western blot analysis. In Figs. 3-5, the protein expression levels of MyD88, TBK1, IRF3, TLR4, TLR3, TRAF6 and NF- $\kappa$ B were increased in normal mice following administration of Concanavalin A compared with the mice in control group without any treatment. However, these expression levels did not increase in HBV transgenic mice following administration of Concanavalin A (Figs. 6-8). The increased expression of MyD88, IRF3, TBK1 and NF- $\mathrm{BB}$ due to immunological hepatitis has been reported previously, which is in agreement with the present findings (17). Furthermore, following administration with Bushen recipe and its disassembled prescriptions, these proteins had no upregulated trend in either normal or HBV groups. NF- $\mathrm{NB}$ further decreased in HBV groups following treatment with Bushen recipe and its disassembled prescriptions (Figs. 6-8). The expression of TRAF6 in QH group and GYL group decreased more notably upon interaction
A

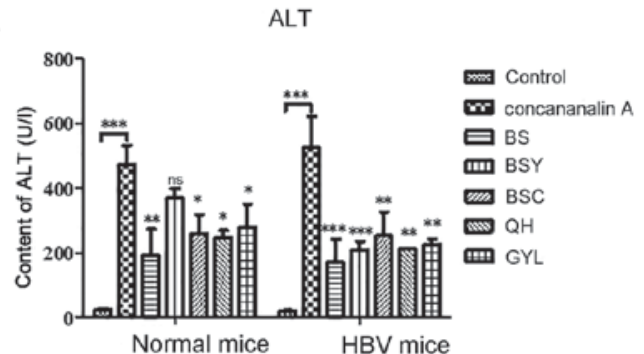

B

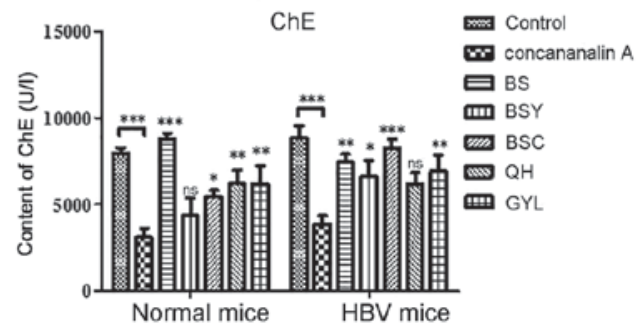

Figure 1. Results of liver function indexes. Mice liver function indexes (A) ALT and (B) ChE in serum were determined. ns indicates no statistical significance, ${ }^{*} \mathrm{P}<0.05,{ }^{* *} \mathrm{P}<0.01,{ }^{* * *} \mathrm{P}<0.001$, vs. the Concanavalin A group or. control group. ALT, Alanine aminotransferase; BS, Bushen recipe; BSC, Bushen-yin; BSY, Bushen-yang; ChE, Cholinesterase; GYL, GanYanLing; HBV, hepatitis B virus; IFN, interferon; IL, interleukin; QH, QingHua; NS, not significant.

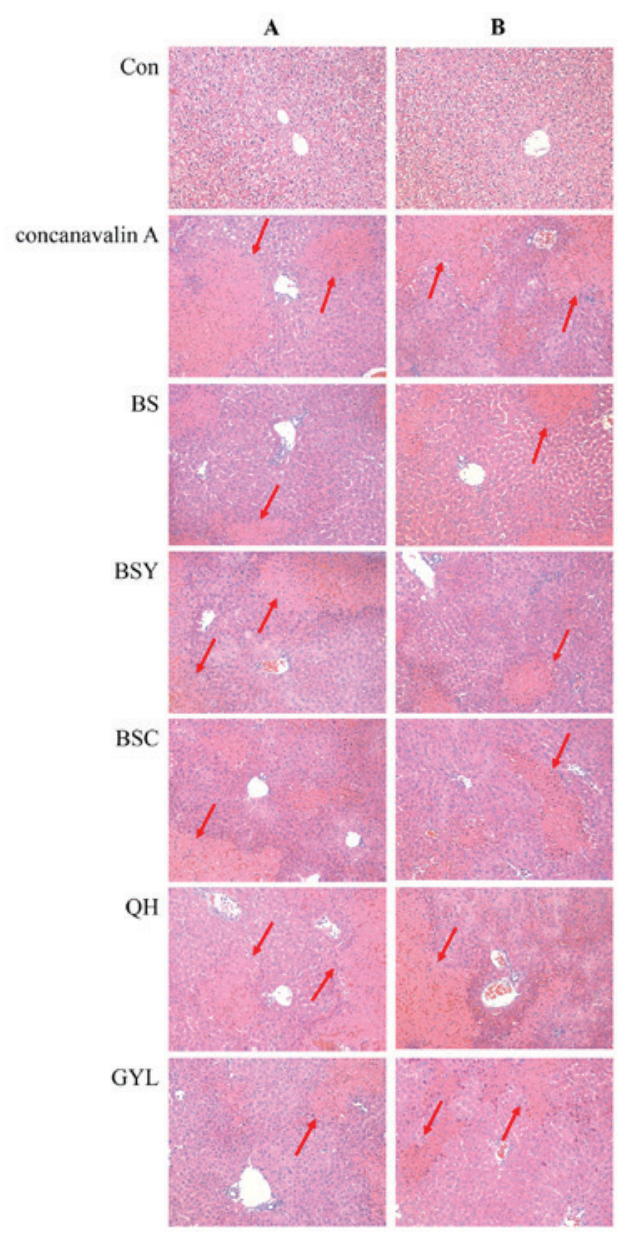

Figure 2. Morphological alterations of liver tissue in mice treated with Concanavalin A, Bushen recipe and its disassembled prescriptions. Liver tissues from (A) normal and (B) HBV transgenic mice were obtained to conduct hematoxylin and eosin staining. $\mathrm{BS}$, Bushen recipe; $\mathrm{BSC}$, Bushen-yin; BSY, Bushen-yang; GYL, GanYanLing; HBV, hepatitis B virus; QH, QingHua. 


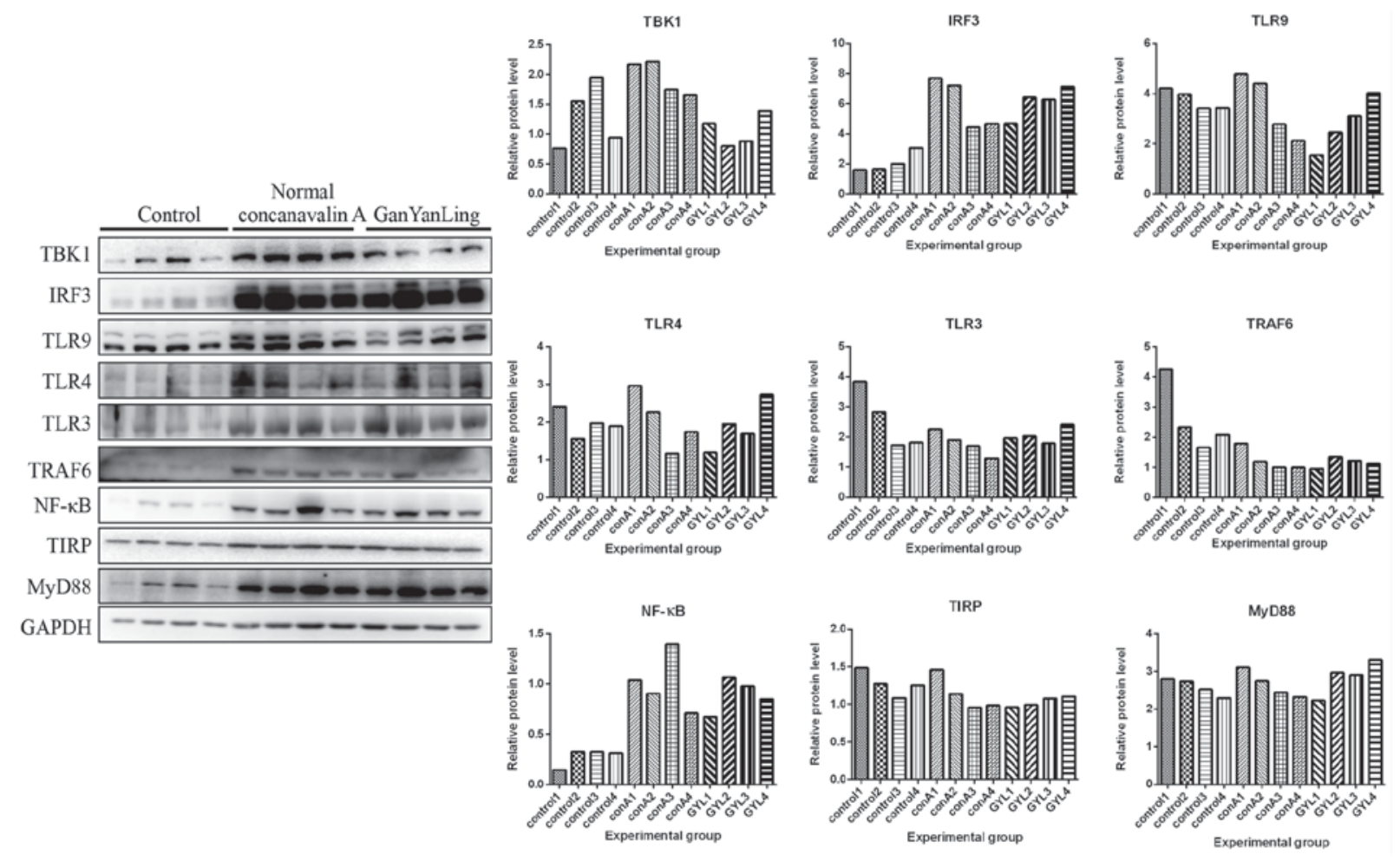

Figure 3. Liver injury in normal mice was induced by Concanavalin A, which were then treated with normal saline (control) and GYL. Liver tissues were obtained to detect protein expression levels of MyD88 (33 kDa), TBK1 (84 kDa), TRAF6 (60 kDa), IRF3 (55 kDa), TLR3 (104 kDa), TLR9 (120 kDa), TLR4 (97-116 kDa), NF-kB (65 kDa) and TIRP (26 kDa) by using western blot analysis. GAPDH was used to confirm the equal amount of protein loaded in each lane. The quantification of the western blot analysis through gray analysis was also performed. conA1-4, mice 1-4 treated with Concanavalin A in the same condition; GYL, GanYanLing; IRF3, interferon regulatory factor 3; MyD88, myeloid differentiation primary response 88; NF-кB, necrosis factor-kB; TBK1, tumor necrosis factor receptor-associated factor-binding kinase; TRAF6, tumor necrosis-associated factor 6; TLR, Toll-like receptor; TIRP, TRAF-interacting protein.

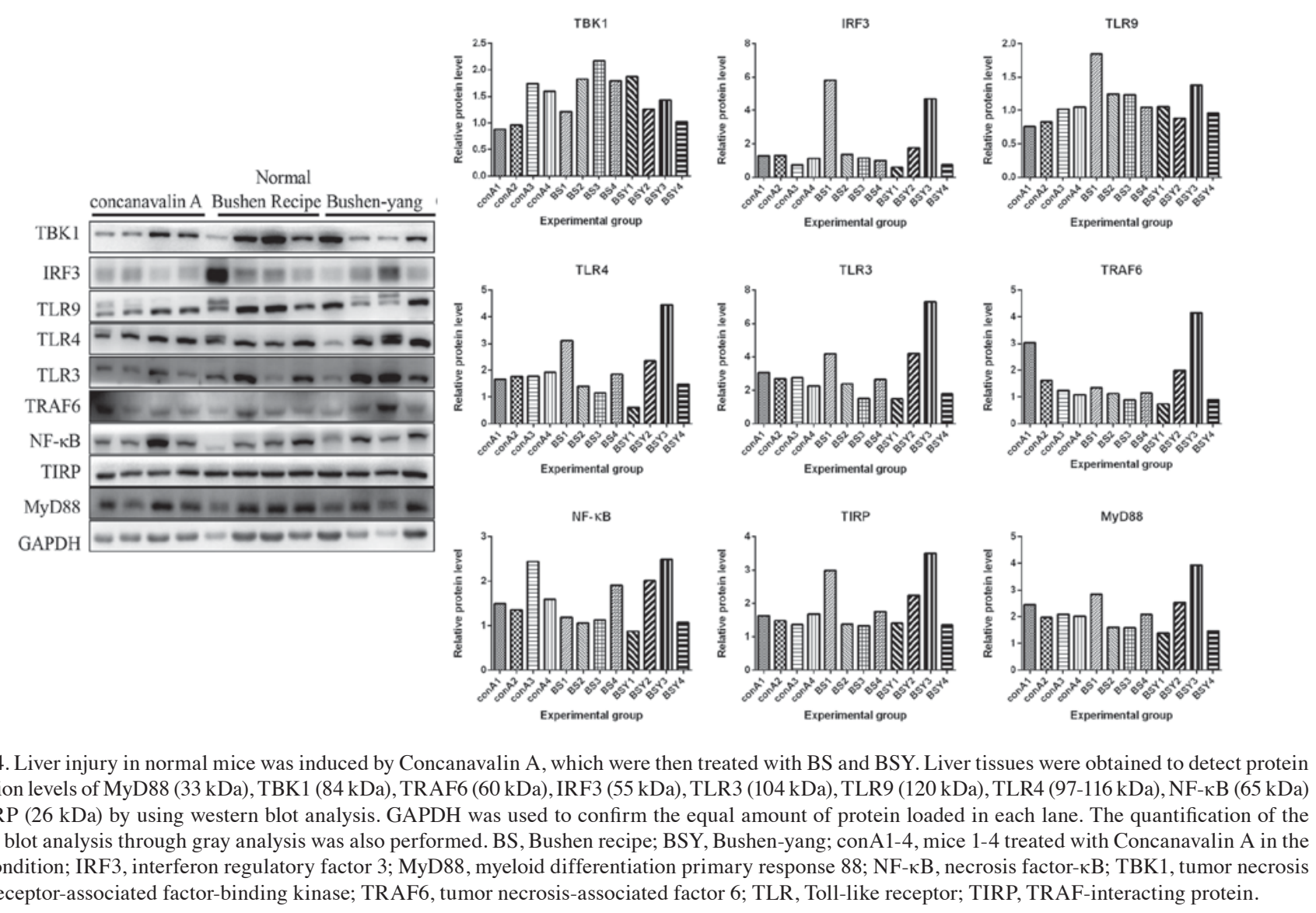



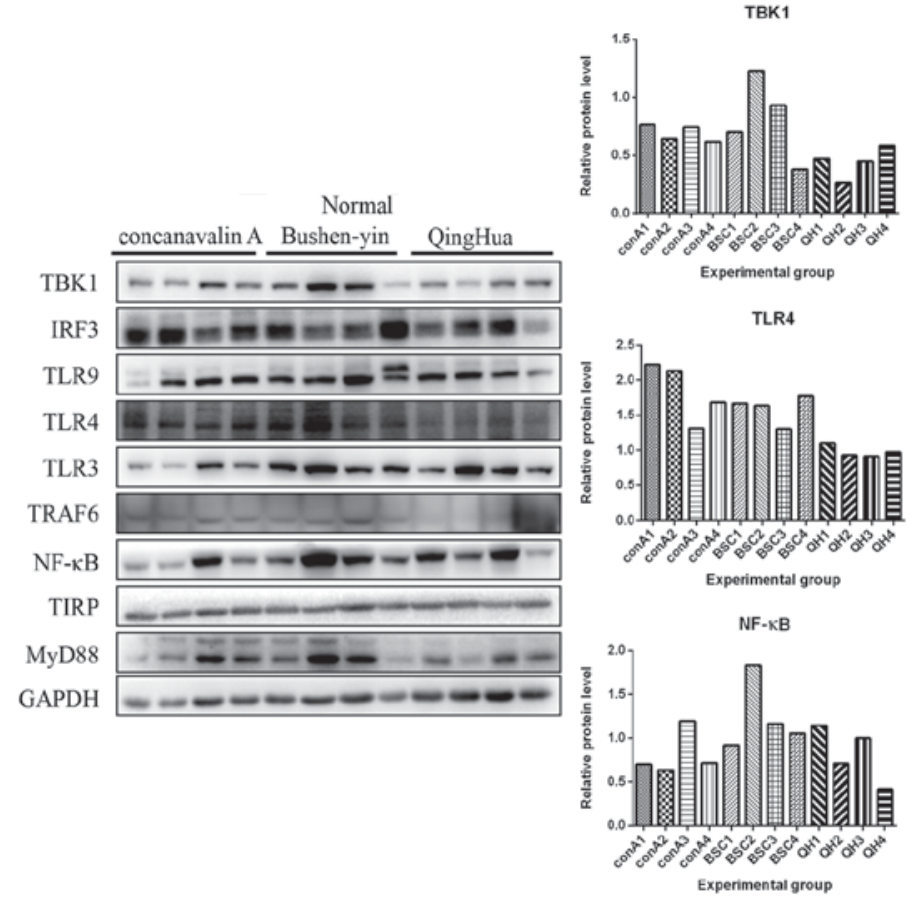
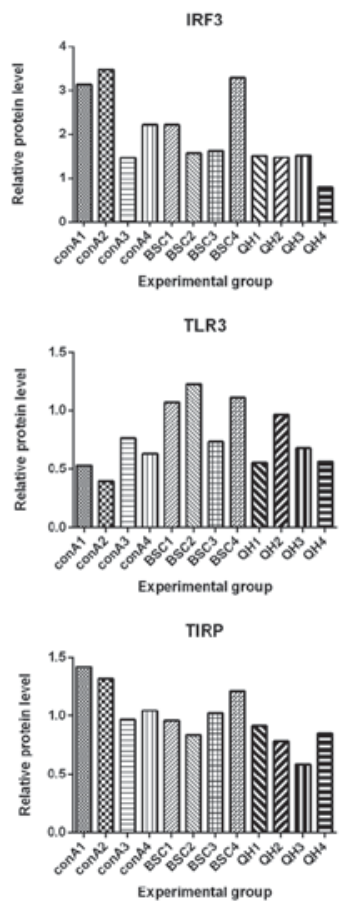
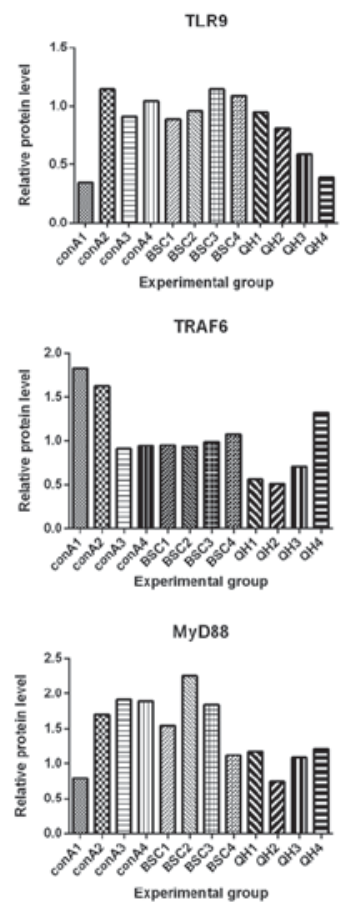

Figure 5. Liver injury in normal mice was induced by Concanavalin A, which were then treated with BSC and QH. Liver tissues were obtained to detect protein expression levels of MyD88 (33 kDa), TBK1 (84 kDa), TRAF6 (60 kDa), IRF3 (55 kDa), TLR3 (104 kDa), TLR9 (120 kDa), TLR4 (97-116 kDa), NF-kB $(65 \mathrm{kDa})$ and TIRP $(26 \mathrm{kDa})$ by using western blot analysis. GAPDH was used to confirm the equal amount of protein loaded in each lane. The quantification of the western blot analysis through gray analysis was also performed. BSC, Bushen-yin; conA1-4, mice 1-4 treated with Concanavalin A in the same condition;

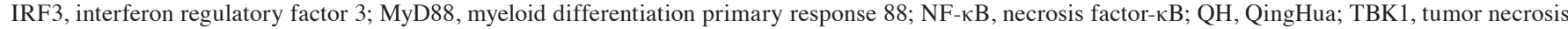
factor receptor-associated factor-binding kinase; TRAF6, tumor necrosis-associated factor 6; TLR, Toll-like receptor; TIRP, TRAF-interacting protein.

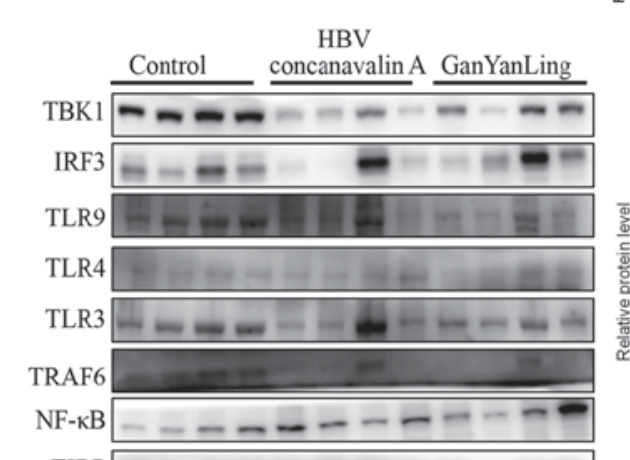

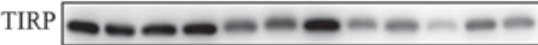

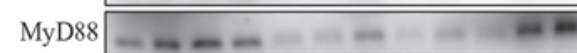

GAPDH $-------\cdots-\infty$
TBK1

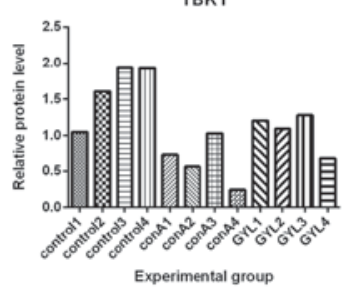

TLR4
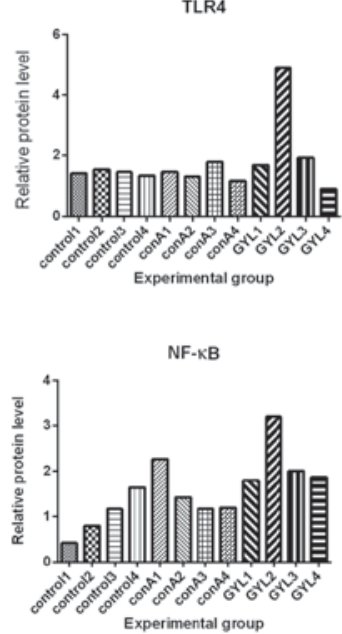

IRF3
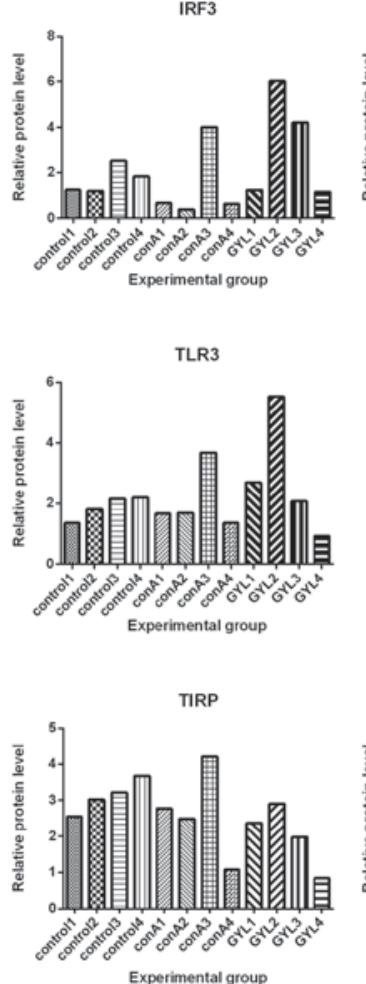

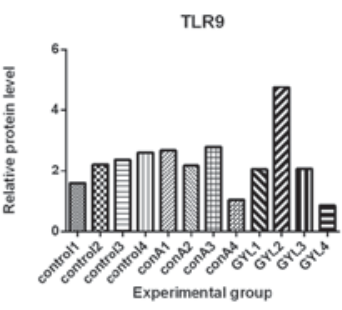

TRAF6
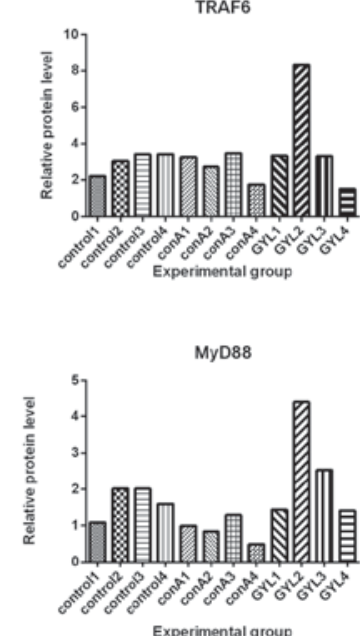

Figure 6. Liver injury in HBV transgenic mice was induced by Concanavalin A, which were then treated with normal saline (control) and GYL. Liver tissues were obtained to detect protein expression levels of MyD88 (33 kDa), TBK1 (84 kda), TRAF6 (60 kDa), IRF3 (55 kDa), TLR3 (104 kDa), TLR9 (120 kDa), TLR4 (97-116 kDa), NF- $\mathrm{KB}(65 \mathrm{kDa})$ and TIRP $(26 \mathrm{kDa})$ by using western blot analysis. GAPDH was used to confirm the equal amount of protein loaded in each lane. The quantification of the western blot analysis through gray analysis was also performed. conA1-4, mice 1-4 treated with Concanavalin A in the same condition; GYL, GanYanLing; HBV, hepatitis B virus; IRF3, interferon regulatory factor 3; MyD88, myeloid differentiation primary response 88; NF-кB, necrosis factor- $\mathrm{BB}$; TBK1, tumor necrosis factor receptor-associated factor-binding kinase; TRAF6, tumor necrosis-associated factor 6; TLR, Toll-like receptor; TIRP, TRAF-interacting protein. 

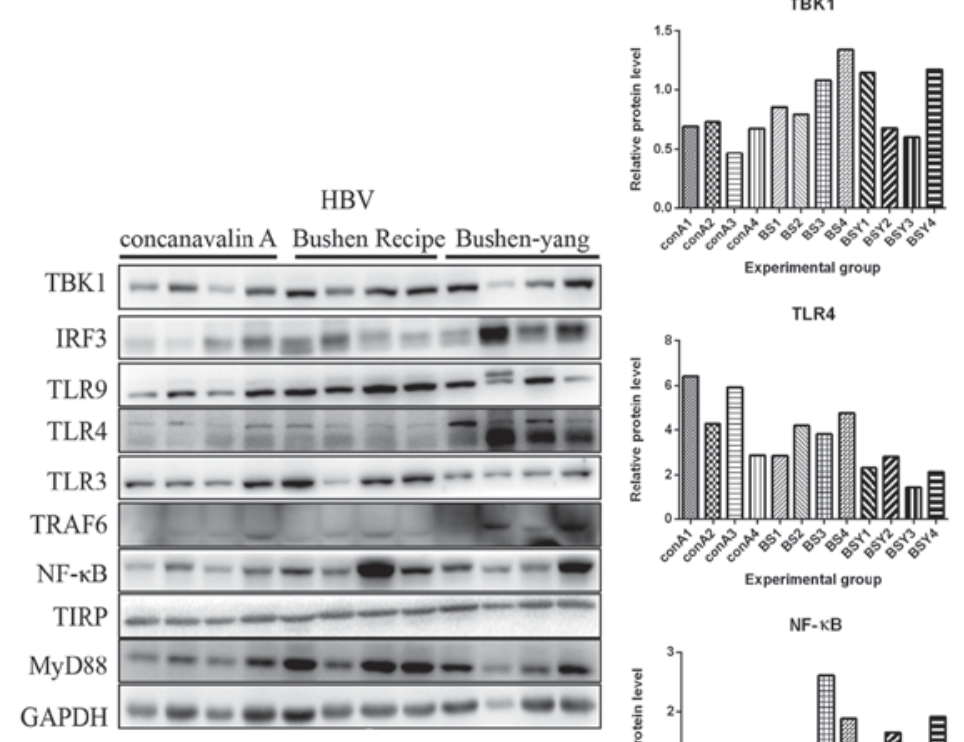

TLR4

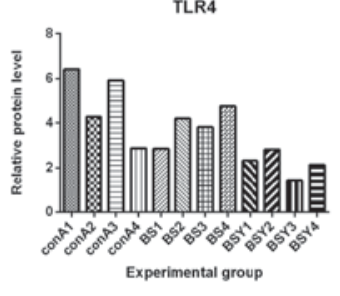

NF- KB

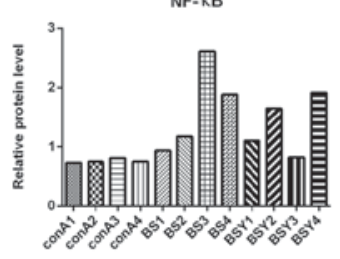

IRF3


TLR9

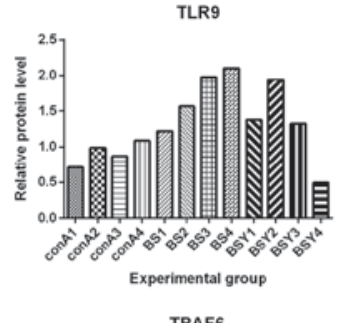

TRAF6
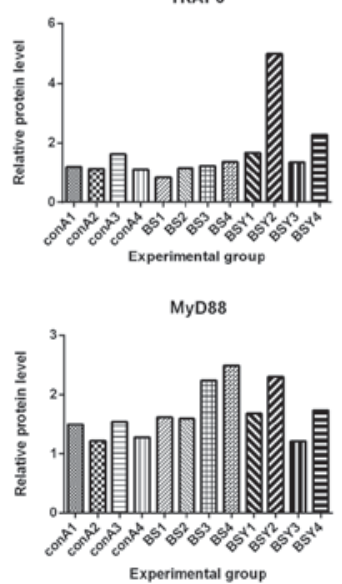

Figure 7. Liver injury in HBV transgenic mice was induced by Concanavalin A, which were then treated with BS and BSY. Liver tissues were obtained to detect protein expression levels of MyD88 (33 kDa), TBK1 (84 kDa), TRAF6 (60 kdD), IRF3 (55 kDa), TLR3 (104 kDa), TLR9 (120 kDa), TLR4 (97-116 kDa), NF-kB $(65 \mathrm{kDa})$ and TIRP $(26 \mathrm{kDa})$ by using western blot analysis. GAPDH was used to confirm the equal amount of protein loaded in each lane. The quantification of the western blot analysis through gray analysis was also performed. conA1-4, mice 1-4 treated with Concanavalin A in the same condition; BS, Bushen recipe; BSY, Bushen-yang; HBV, hepatitis B virus; IRF3, interferon regulatory factor 3; MyD88, myeloid differentiation primary response 88; NF- $\mathrm{BB}$, necrosis factor-кB; TBK1, tumor necrosis factor receptor-associated factor-binding kinase; TRAF6, tumor necrosis-associated factor 6; TLR, Toll-like receptor; TIRP, TRAF-interacting protein.
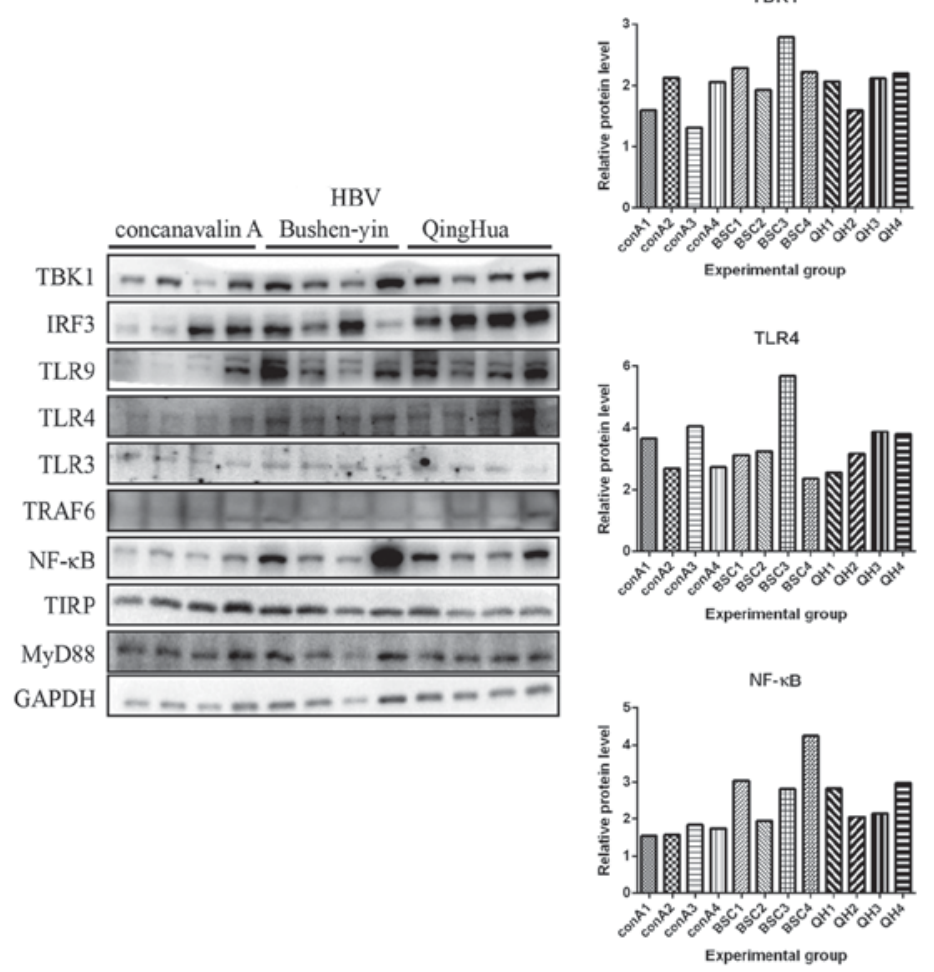
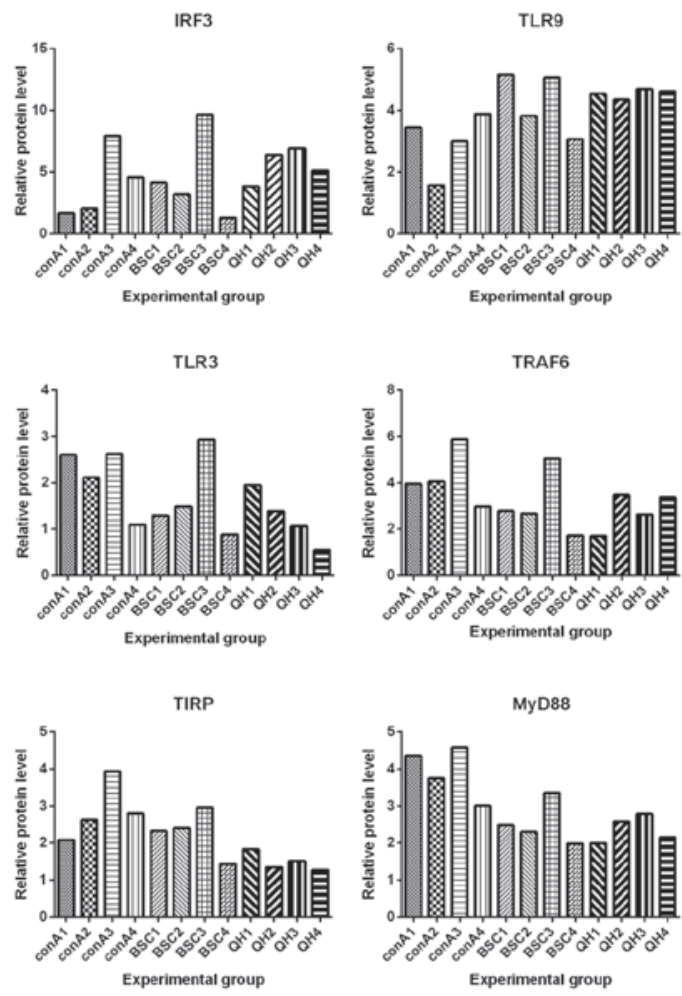

Figure 8. Liver injury in HBV transgenic mice was induced by Concanavalin A, which were then treated with BSC and QH. Liver tissues were obtained to detect protein expression levels of MyD88 (33 kDa), TBK1 (84 kDa), TRAF6 (60 kDa), IRF3 (55 kDa), TLR3 (104 kDa), TLR9 (120 kDa), TLR4 (97-116 kDa), NF-kB $(65 \mathrm{kDa})$ and TIRP $(26 \mathrm{kDa})$ by using western blot analysis. GAPDH was used to confirm the equal amount of protein loaded in each lane. The quantification of the western blot analysis through gray analysis was also performed. BSC, Bushen-yin; conA1-4, mice 1-4 treated with Concanavalin A in the same condition; QH, QingHua; IRF3, interferon regulatory factor 3; MyD88, myeloid differentiation primary response 88; NF- $\mathrm{B}$, necrosis factor- $\mathrm{kB}$; TBK1, tumor necrosis factor receptor-associated factor-binding kinase; TRAF6, tumor necrosis-associated factor 6; TLR, Toll-like receptor; TIRP, TRAF-interacting protein. 



Figure 9. Expression levels of inflammatory factors in all mice treated with Concanavalin A, BS and its disassembled prescriptions. Expression levels of (A) IL-6, (B) IFN- $\gamma$ and (C) TNF- $\alpha$ and (D) IL-1 as measured by ELISA. ns indicates no statistical significance, ${ }^{*} \mathrm{P}<0.05,{ }^{* *} \mathrm{P}<0.01,{ }^{* * * *} \mathrm{P}<0.001$, vs. the Concanavalin A group or control group. BS, Bushen recipe; BSC, Bushen-yin; BSY, Bushen-yang; GYL, GanYanLing; HBV, hepatitis B virus; IFN, interferon; IL, interleukin; QH, QingHuang; TNF, tumor necrosis factor; NS, not significant.

of corresponding treatment. Additionally, compared with the control group, the expression levels of MyD88, TBK1, TRAF6, IRF3, TLR3, TLR9 and TIRP were decreased to varying extents, indicating the pharmaceutical effect of Bushen recipe and all its disassembled prescriptions. In conclusion, these results indicated that the TLR3/9 signaling pathway may be inhibited by Bushen Recipe and its disassembled prescriptions to repair liver injury induced by Concanavalin $\mathrm{A}$.

Bushen recipe and its disassembled prescriptions decrease the Concanavalin A-induced increase of various inflammatory factors expression. IL-6, TNF- $\alpha$ and IL- 1 are primary inflammatory factors that affect the downstream pathway of NF- $\mathrm{kB}$, and IFN- $\gamma$ affects the downstream pathway of IRF3 $(18,19)$. These inflammatory factors were detected by ELISA as demonstrated in Fig. 9. The expression levels of IL-6, IL-1, TNF- $\alpha$ and IFN- $\gamma$ were significantly increased in normal and HBV mice following injection with Concanavalin A, compared with in the control group. However, following treatment with Bushen recipe and its disassembled prescriptions, the Concanavalin A-induced upregulated expression levels of IL-6, IL-1 and TNF- $\alpha$ decreased significantly in the BS group, while only IL-1 in BSY group decreased significantly and only IL-6 in the BSC and QH group decreased significantly. These results are consistent with activation state of NF- $\kappa B$ in western blot analysis, and the downstream pathway of NF- $\kappa \mathrm{B}$ requires further investigation and validation. Furthermore, the increase of IFN- $\gamma$ expression was evident following Concanavalin A injection in normal and HBV mice, while the content of IFN- $\gamma$ in the mice treated with the Bushen recipe and its disassembled prescriptions decreased only slightly in BSC and BSY groups. Therefore, the results of the present study suggested that the Bushen recipe and its disassembled prescriptions (BSC and BSY in particular) may repair liver injury induced by Concanavalin A by inhibiting the expression of inflammatory factors. Variations in the effects exhibited by Bushen recipe and its disassembled prescription may be due to the differing compunds present; however further research is required.

\section{Discussion}

Hepatitis B has become one of the most prevalent and serious infectious diseases in the world and persistently infects 350 million people worldwide with the added risk of development of other chronic liver diseases (20-22). It not only seriously affects human health, but it also brings a vast economic burden to patients and society.

During acute HBV infection, viral clearance could be achieved in a non-cytolytic manner. This process is mediated by inflammatory cytokines such as IFN- $\alpha$, IFN- $\beta$, IFN- $\gamma$ and TNF- $\alpha$ which are produced by activated T lymphocytes, natural killer cells or antigen nonspecific macrophages (23-25). IFN- $\gamma$ inhibits HBV replication by disturbing the formation of viral RNA-containing capsids or promoting their degradation; both of these processes require proteasome activity (26-28). The mechanism of the TNF- $\alpha$-induced antiviral response remains unclear. It has been reported that TNF- $\alpha$ blocks the formation or stability of cytoplasmic viral capsids via activation of NF- $\mathrm{B}$, however the antiviral effector genes remain unknown $(29,30)$. Therefore, herein, HBV mice were used to initiate liver injury associated with Con A in order to study the effects of Con A on liver injury.

Current treatment methods including vaccines, immunomodulators, interferons and nucleoside analogs are not satisfactory. Traditional Chinese Medicines (TCMs) with 
various components and multiple targets may have advantages in treating HBV infection (31). The effect of TCMs on serum HBs Ag of patients with chronic hepatitis B has been systematically studied (32-34). Notably, the Bushen recipe used in this study was prepared according to the formula of 'Bushen Granule' (Shanghai Pharmacy), which means that the recipe is unique. An additional Bushen recipe with different ingredients, which is also produced by our hospital, Shuguang Hospital (Shanghai, China), was reported to reduce both the number of Th1/Th2 cells infiltrating the liver and serological factors associated with liver function, alleviating pathological alterations of the liver (35).

It is reported that the production of inflammatory factors is associated with the TLR3/9 signaling pathway $(36,37)$. A particular subgroup within the TLR family including TLR3, TLR7, TLR8, and TLR9 is localized in endosomes and recognizes nucleic acids such as viral DNA or RNA. The other subgroup of surface-expressed TLR1, TLR2, TLR4, TLR5, and TLR6 recognizes components of the extracellular bacterial and fungal cell wall, as well as some viral proteins $(38,39)$. Binding of TLR agonists to their receptors initiates the activation of complex networks of intracellular signaling transduction pathways to coordinate the inflammatory response. Conformational changes and dimerization of TLRs occur upon binding with ligands. The important components in these signaling networks are the adaptor proteins and several protein kinases including extracellular signal-regulated kinase, c-Jun $\mathrm{N}$-terminal kinase, p38, mitogen-activated protein kinase, and phosphoinositide 3-kinase, and the transcription factors IRF3/5/7, NF- $\kappa \mathrm{B}$, and activator protein-1. The activation of these transcription factors leads to the induction of type I IFNs, pro-inflammatory cytokines, or co-stimulatory molecules, which are involved in antiviral responses $(40,41)$. The crucial adaptor proteins, such as MyD88 are employed by almost all TLRs except TLR3; TIRAP is used by TLR 2 and TLR4, TRIF is used by TLR3 and TLR4, and TRAM is employed only by TLR4 (42). The results of the present study revealed that Bushen recipe and its disassembled prescriptions may inhibit the activation of TLR3/9 signaling pathway, particularly $\mathrm{NF}-\kappa \mathrm{B}$ downstream. Combined with ELISA results, it was observed that Bushen recipe and its disassembled prescriptions decreased the expression of NF- $\kappa \mathrm{B}$ and its downstream inflammatory factors, including TNF- $\alpha$, IL-6 and IL-1; however future studies are required to confirm these results.

In conclusion, in the present study, the combination of the measurement of ALT and ChE, which are both synthesized in liver and could be used to represent the degree of liver injury, and the microscopic observation of Hematoxylin and Eosin Stained liver tissues displayed that Bushen recipe and its disassembled prescriptions could repair liver injury caused by Concanavalin A. Furthermore, investigations verified that the capability of Bushen recipe and its disassembled prescriptions results from the inhibition of TLR3/9 signaling pathway and associated expression of inflammatory factors. Notably, the present study may provide a novel strategy for the development of anti-HBV drugs in the future.

\section{Acknowledgements}

Not applicable.

\section{Funding}

The present study was supported by the National Natural Science Foundation of China (grant no. 81373618).

\section{Availability of data and materials}

The datasets used and/or analyzed during the current study are available from the corresponding author on reasonable request.

\section{Authors' contributions}

HN and YG made substantial contributions to the concept and design of the present study. HN, ZM and BZ conducted the experiments. JC, LW and RW conducted data analysis. HN, LW and RW produced the manuscript. All authors read and approved the final manuscript.

\section{Ethics approval and consent to participate}

All animal experiments were carried out according to the regulations of and approved by Laboratory Animal Administration of Shanghai University of Traditional Chinese Medicine.

\section{Consent for publication}

Not applicable.

\section{Competing interests}

Authors declare that they have no competing interests.

\section{References}

1. Tiollais P, Pourcel C and Dejean A: The hepatitis B virus. Nature 317: 489-495, 1985.

2. Minor MM and Slagle BL: Hepatitis B virus HBx protein interactions with the ubiquitin proteasome system. Viruses 6: 4683-4702, 2014.

3. de Franchis R, Hadengue A, Lau G, Lavanchy D, Lok A, McIntyre N, Mele A, Paumgartner G, Pietrangelo A, Rodés J, et al: EASL International Consensus Conference on Hepatitis B. 13-14 September, 2002 Geneva, Switzerland. Consensus statement (long version). J Hepatol 39 (Suppl 1): S3-S25, 2003

4. Inchauspé G and Michel ML: Vaccines and immunotherapies against hepatitis B and hepatitis C viruses. J Viral Hepat 14 (Suppl 1): S97-S103, 2007.

5. Lu M, Menne S, Yang D, Xu Y and Roggendorf M: Immunomodulation as an option for the treatment of chronic hepatitis B virus infection: Preclinical studies in the woodchuck model. Expert Opin Invest Drugs 16: 787-801, 2007.

6. Michel ML and Tiollais P: Hepatitis B vaccines: Protective efficacy and therapeutic potential. Pathol Biol (Paris) 58: 288-295, 2010.

7. Roggendorf M and Lu M: Woodchuck Hepatitis Virus. 2007.

8. Garcia ML: Regulation of hepatitis B virus replication by the ubiquitin-proteasome pathway (unpublished $\mathrm{PhD}$ thesis). Yale University School of Medicine, New Haven, CT, 2010.

9. Xiao X, Zhao P, Rodriguez-Pinto D, Qi D, Henegariu O, Alexopoulou L, Flavell RA, Wong FS and Wen L: Inflammatory regulation by TLR3 in acute hepatitis. J Immunol 183: 3712-3719. 2009.

10. Keil JM, Shen Z, Briggs SP and Patrick GN: Regulation of STIM1 and SOCE by the ubiquitin-proteasome system (UPS). PLoS One 5: e13465, 2010.

11. Cacciapuoti F: Role of ubiquitin-proteasome system (UPS) in left ventricular hypertrophy (LVH). Am J Cardiovasc Dis 4: 1-5, 2014. 
12. Mata-Cantero L, Lobato-Gil S, Aillet F, Lang V and Rodriguez MS: The Ubiquitin-Proteasome System (UPS) as a cancer drug target: Emerging mechanisms and therapeutics. In: Stress Response Pathways in Cancer: From Molecular Targets to Novel Therapeutics. Wondrak GT (ed.) Springer Netherlands, Dordrecht, pp225-264, 2015.

13. Gao YQ, Yao Y and Li M: Effect of bushen recipe on the immune effector molecules of natural killer cells in patients with chronic hepatitis B. Zhongguo Zhong Xi Yi Jie He Za Zhi 30: 710-713, 2010 (In Chinese)

14. Chen XF, Chen SH, Gui-Yuan L, Xiao LW and Lou ZH: Research and discussion on mechanism of treating osteoporosis by Bushen Huoxue Recipe. Chin Tradit Pat Med 33: 1130-1134, 2011.

15. Tan Y, Tan L, Huang S, Lu J and Yu L: Content determination of active component in Huangqi Yinyanghuo group and its effects on hTERT and Bcl-2 protein in osteosarcoma. J Anal Methods Chem 2014: 769350, 2014.

16. Luo HY: Clinical research of using Bushen Huoxue prescription in the treatment of early diabetic nephropathy. Sci Technol Eng 8: 2176-2179, 2008

17. Zhang E and Lu M: Toll-like receptor (TLR)-mediated innate immune responses in the control of hepatitis B virus (HBV) infection. Med Microbiol Immunol 204: 11-20, 2015.

18. Cortez M, Carmo LS, Rogero MM, Borelli P and Fock RA: A high-fat diet increases IL-1, IL- 6 , and TNF- $\alpha$ production by increasing NF- $\kappa \mathrm{B}$ and attenuating PPAR- $\gamma$ expression in bone marrow mesenchymal stem cells. Inflammation 36: 379-386, 2013

19. Guinn Z, Brown DM and Petro TM: Activation of IRF3 contributes to IFN- $\gamma$ and ISG54 expression during the immune responses to B16F10 tumor growth. Int Immunopharmacol 50 121-129, 2017.

20. Coluccio C, Begini P, Marzano A, Pellicelli A, Imperatrice B, Anania G, Delle Fave G and Marignani M: Hepatitis B in patients with hematological diseases: An update. World J Hepatol 9: 1043-1053, 2017.

21. Stanley M: Tumour virus vaccines: Hepatitis B virus and human papillomavirus. Philos Trans R Soc Lond B Biol Sci 372: $20160268,2017$.

22. Stasi C, Silvestri C, Voller F: Emerging trends in epidemiology of hepatitis B virus infection. J Clin Transl Hepatol 5: 272-276, 2017.

23. Guidotti LG, Ishikawa T, Hobbs MV, Matzke B, Schreiber R and Chisari FV: Intracellular inactivation of the hepatitis B virus by cytotoxic T lymphocytes. Immunity 4: 25-36, 1996.

24. Guidotti LG, Rochford R, Chung J, Shapiro M, Purcell R and Chisari FV: Viral clearance without destruction of infected cells during acute HBV infection. Science 284: 825-829, 1999.

25. Guidotti LG and Chisari FV: Noncytolytic control of viral infections by the innate and adaptive immune response. Annu Rev Immunol 19: 65-91, 2001.

26. Robek MD, Wieland SF and Chisari FV: Inhibition of hepatitis $\mathrm{B}$ virus replication by interferon requires proteasome activity. J Virol 76: 3570-3574, 2002.
27. Wieland SF, Eustaquio A, Whitten-Bauer C, Boyd B and Chisari FV: Interferon prevents formation of replicationcompetent hepatitis B virus RNA-containing nucleocapsids. Proc Natl Acad Sci USA 102: 9913-9917, 2005.

28. Xu C, Guo H, Pan XB, Mao R, Yu W, Xu X, Wei L, Chang J, Block TM and Guo JT: Interferons accelerate decay of replication-competent nucleocapsids of hepatitis B virus. J Virol 84: 9332-9340, 2010

29. Biermer M,Puro R and Schneider RJ: Tumor necrosis factor alpha inhibition of hepatitis B virus replication involves disruption of capsid Integrity through activation of NF-kappaB. J Virol 77: 4033-4042, 2003.

30. Puro R and Schneider RJ: Tumor necrosis factor activates a conserved innate antiviral response to hepatitis B virus that destabilizes nucleocapsids and reduces nuclear viral DNA. J Virol 81: 7351-7362, 2007.

31. Chen JJ: The Anti-HBV constituents from traditional chinese medicine of swertia mileensis. Planta Med 79: OP23, 2013

32. Dai XQ, Cai WT, Wu X, Chen Y and Han FM: Protocatechuic acid inhibits hepatitis $B$ virus replication by activating ERK1/2 pathway and down-regulating $\mathrm{HNF} 4 \alpha$ and $\mathrm{HNF} 1 \alpha$ in vitro. Life Sci 180: 68-74, 2017.

33. Yang Y, Jiang HY, Shi Y, He JL, Su S and Chen Z: Chinese herbal medicine for carriers of the hepatitis B virus: An updated systematic review and meta-analysis. Pharmazie 69: 723-730, 2014.

34. Dai JJ, Tao HM, Min QX and Zhu QH: Anti-hepatitis B virus activities of friedelolactones from Viola diffusa Ging. Phytomedicine 22: 724-729, 2015.

35. Qiao B, Zhou ZH, Man LI, Sun XH, Jin SG, Zhu XJ, Huang LY, Zhang X and Gao YQ: Experimental study of Bushen Recipe on Th1/Th2 in liver tissue of chronic hepatic injury mice model induced by ConA. China J Tradit Chin Med Pharm 32: 841-844, 2017 (In Chinese)

36. Xue D, Ma Y, Li M, Li Y, Luo H, Liu X and Wang Y: Mycoplasma ovipneumoniae induces inflammatory response in sheep airway epithelial cells via a MyD88-dependent TLR signaling pathway. Vet Immunol Immunopathol 163: 57-66, 2015.

37. Li X, Wang S, Zhu R, Li H, Han Q and Zhao RC: Lung tumor exosomes induce a pro-inflammatory phenotype in mesenchymal stem cells via NFkB-TLR signaling pathway. J Hematol Oncol 9: 42, 2016.

38. Gay NJ and Gangloff M: Structure and function of Toll receptors and their ligands. Annu Rev Biochem 76: 141-165, 2007.

39. Takeda K, Kaisho T and Akira S: Toll-like receptors. Annu Rev Immunol 21: 335-376, 2003.

40. Schwabe RF, Seki E and Brenner DA: Toll-like receptor signaling in the liver. Gastroenterology 130: 1886-1900, 2006.

41. Seki E and Brenner DA: Toll-like receptors and adaptor molecules in liver disease: Update. Hepatology 48: 322-335, 2008.

42. Lee MS and Kim YJ: Signaling pathways downstream of pattern-recognition receptors and their cross talk. Annu Rev Biochem 76: 447-480, 2007. 